\title{
Studies on Aspirin Crystals Generated by a Modified Vapor Diffusion Method
}

\author{
Amit Mittal, ${ }^{1}$ Deepak Malhotra, ${ }^{1}$ Preeti Jain, ${ }^{1}$ Anupama Kalia, ${ }^{1}$ and Tamilvanan Shunmugaperumal ${ }^{1,2}$
}

Received 30 June 2015; accepted 8 October 2015; published online 16 October 2015

Abstract. The objectives of the current investigation were (1) to study the influence of selected two
different non-solvents (diethylether and dichloromethane) on the drug crystal formation of a model drug,
aspirin (ASP-I) by the modified vapor diffusion method and (2) to characterize and compare the
generated crystals (ASP-II and ASP-III) using different analytical techniques with that of unprocessed
ASP-I. When compared to the classical vapor diffusion method which consumes about 15 days to generate
drug crystals, the modified method needs only $12 \mathrm{~h}$ to get the same. Fourier transform-infrared spectros-
copy (FT-IR) reveals that the internal structures of ASP-II and ASP-III crystals were identical when
compared with ASP-I. Although the drug crystals showed a close similarity in X-ray diffraction patterns,
the difference in the relative intensities of some of the diffraction peaks (especially at $2 \theta$ values of around
7.7 and 15.5) could be attributed to the crystal habit or crystal size modification. Similarly, the differential
scanning calorimetry (DSC) study speculates that only the crystal habit modifications might occur but
without involving any change in internal structure of the generated drug polymorphic form I. This is
further substantiated from the scanning electron microscopy (SEM) pictures that indicated the formation
of platy shape for the ASP-II crystals and needle shape for the ASP-III crystals. In addition, the observed
slow dissolution of ASP crystals should indicate polymorph form I formation. Thus, the modified vapor
diffusion method could routinely be used to screen and legally secure all possible forms of other drug
entities too.

KEYWORDS: aspirin; crystal habit; crystallization; scanning electron microscopy; vapor diffusion.

\section{INTRODUCTION}

It has long been known that a crystalline active pharmaceutical ingredient (API) consists of well-defined external and internal structures. Whereas the internal structure refers to the molecular arrangement in the crystal lattice which deals with the polymorphic forms, the external structure concurs to the crystal habit describing either outer morphology or crystal shape but at the same time, without being involved in the change of the API's internal structure (1). Though a single internal structure with different external morphologies often exists for the particular API, vice versa however is rarely evident. Nevertheless, the change in external and internal structures of the API within dosage form itself should affect not only the chemical and physical stabilities but also the performance of the dosage forms which includes dissolution, flowability, tableting, and syringeability (2-4). Since there is no single reliable method that existed to be able to legally secure all possible drug forms, it becomes therefore necessary for both innovator and generic drug companies to make every effort to find new and efficient (in terms of time and/or resources) screening methods. For instance, in the watering out

\footnotetext{
${ }^{1}$ Lovely School of Pharmaceutical Sciences, Lovely Professional University, Jalandhar-Delhi, G.T. Road (NH-1), Phagwara, Jalandhar, Punjab, India144411.

${ }^{2}$ To whom correspondence should be addressed. (e-mail: tamilvanan1@yahoo.co.in)
}

technique, the API solution prepared at higher temperature is added to water maintained at lower temperature $\left(3^{\circ} \mathrm{C}\right)$ while in the salting out method, the crystal is precipitated from the API solution by the addition of non-solvent $(5,6)$. Moreover, the use of various additives like PVP, PEG, PVP K30, or Tween 80 and the presence of certain impurities like thymine and thymidine in the crystallizing media can also influence the API crystal habit (7-9). Furthermore, kinetically controlled fast evaporation of solvent from solutions, either by nitrogen gas flow or by rotary evaporation, also helps to identify rapidly the various forms of APIs $(10,11)$.

An alternative but not yet well-documented way to produce the API crystal of high purity and desirable habits is a classical vapor diffusion method (Fig. 1a). Here, a small container containing the API solution made from a solvent is placed into a larger vessel containing the small amount of miscible and volatile nonsolvent. The whole setup is then kept in a desiccator over many days (about 12 days) for the API crystals to form (12). In the current investigation, a modification has been proposed for the desiccator-based classical vapor diffusion method. Typically, the desiccator is being replaced by a two-beaker assembly (Fig. 1b). A bigger-sized beaker is taken and covered with an airtight lid having a hole to insert a thermometer. About one fourth or one eighth volume of the bigger-sized beaker is filled with the selected non-solvent. A heating mantle is also fitted at the bottom of the bigger-sized beaker in order to force the evaporation of the nonsolvent. A small-sized beaker containing the saturated solution of API in acetonitrile is kept inside the bigger-sized beaker. Upon 
a

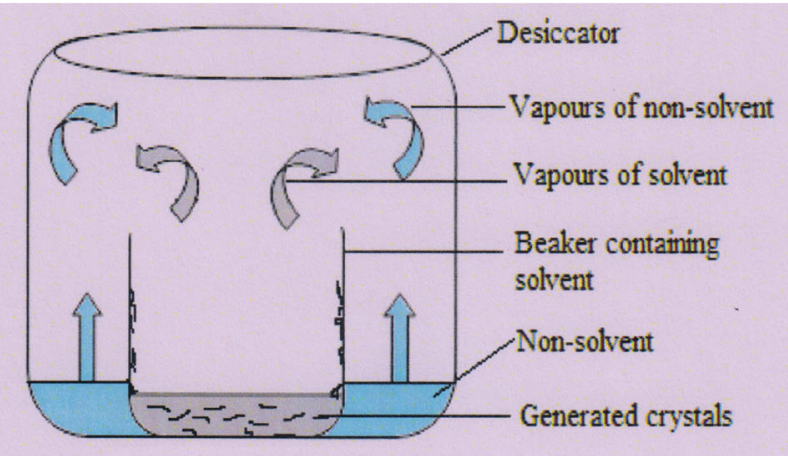

b

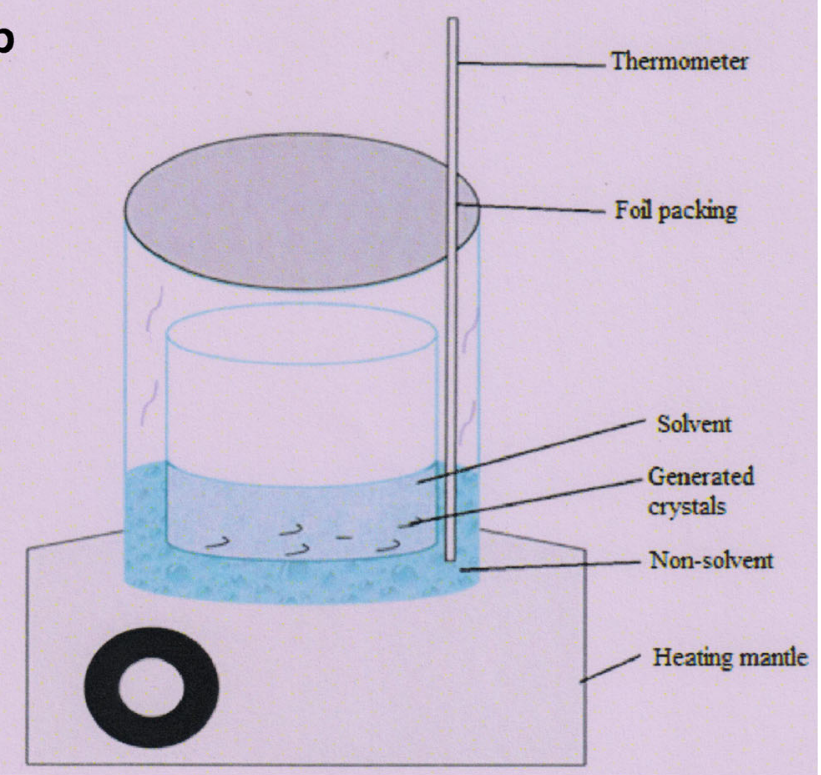

Fig. 1. a Pictorial representation of classical vapor diffusion method (adapted from Guillory 1999 (12)). b Pictorial representation of the developed modified vapor diffusion method

heating, the non-solvent vapor enters at a controlled manner into the small-sized beaker. This two-beaker assembly is likely to enhance the miscibility of non-solvent with the solvent of the API saturated solution. Subsequently, the miscibility of nonsolvent with solvent leads to the formation of API crystal relatively at a shorter experimental duration in comparison to the classical vapor diffusion method. Aspirin (ASP-I) and dichloromethane (DCM) or diethylether (DEE) were chosen as the model drug and non-solvents, respectively.

The objectives of the current investigation were (1) to study the influence of the selected two different non-solvents (DEE and DCM) on the crystal formation of a model drug, aspirin (ASP-I), by the modified vapor diffusion method and (2) to characterize and compare the generated crystals (ASPII and ASP-III) using different analytical techniques with that of unprocessed ASP-I.

\section{MATERIALS AND METHODS}

\section{Materials}

Aspirin (ASP-I) was obtained as an ex-gratis from Jackson Laboratories Pvt. Ltd., Amritsar. India. Diethyl ether was purchased from Qualikem Fine Chem Pvt. Ltd., New Delhi, India.

Acetonitrile and dichloromethane were purchased from Central Drug House Pvt. Ltd., New Delhi, India. All the other reagents were of analytical grade. Triple distilled water was used wherever required.

\section{METHODS}

\section{Aspirin Crystal Formation Induced by Diethylether}

One gram of ASP-I was accurately weighed and dissolved in $5 \mathrm{ml}$ acetonitrile in a $50-\mathrm{ml}$ capacity beaker to prepare a supersaturated solution. This beaker was then placed in a 250$\mathrm{ml}$ capacity beaker which was already filled with $30 \mathrm{ml}$ DEE, a non-solvent. Extreme care was taken so that the walls of both the beakers are not touching each other as shown in Fig. 1b. The whole setup was tightly closed from the top using aluminum foil, and heated at a temperature of $35^{\circ} \mathrm{C}$. After complete evaporation of DEE from the 250-ml beaker, the solution that remained in the $50-\mathrm{ml}$ beaker was then placed in a refrigerator $\left(3-4^{\circ} \mathrm{C}\right)$ for $12 \mathrm{~h}$ to obtain the ASP-II crystals. The obtained crystals were washed with DEE, filtered, and then were allowed to dry in a hot air oven at $40^{\circ} \mathrm{C}$ for $10 \mathrm{~min}$.

\section{Aspirin Crystal Formation Induced by Dichloromethane}

Instead of using the $30 \mathrm{ml}$ of DEE as the non-solvent and the heating temperature of $35^{\circ} \mathrm{C}, 60 \mathrm{ml}$ of $\mathrm{DCM}$ and heating temperature of $40^{\circ} \mathrm{C}$ were used in order to produce another form of aspirin crystal (ASP-III), while other conditions remained the same as that of the above said procedure.

\section{Fourier Transform-Infrared (FT-IR) Spectroscopy}

The IR spectra of ASP-I, ASP-II, and ASP-III were obtained using a FT-IR spectrophotometer 8400S (Shimadzu, Japan) following the sample preparation with dried $\mathrm{KBr}$ powder. All the spectra were recorded in the spectral region of $500-4000 \mathrm{~cm}^{-1}$.

\section{Powder X-ray Diffraction (PXRD)}

The X-ray diffractograms of ASP-I, ASP-II, and ASP-III were obtained by using an X-ray diffractometer (PAN analytical's X' Pert PRO, The Netherlands), equipped with X' Pert PRO data collector software. The radiations used were generated by $\mathrm{CuK} \alpha^{-1}$ source fitted with a filter made up of nickel metal, and the instrument was operated at a voltage of $45 \mathrm{KV}$. The drug crystals were packed into the holder and gently pressed by the glass slide to ensure the co-planarity between the samples and the surface of the sample holder. Samples were scanned for $2 \theta$ values over the range of $5-45^{\circ}$ at a scanning rate of $10 \% \mathrm{~min}$ to get the X-ray diffractograms.

\section{Differential Scanning Calorimetry (DSC)}

DSC thermograms of ASP-I, ASP-II, and ASP-III were recorded using a differential scanning calorimeter (Diamond DSC Perkin, USA). Samples (0.5 to $3.0 \mathrm{mg}$ ) were heated (temperature range $20-160^{\circ} \mathrm{C}$ ) in sealed aluminum pans 
(empty aluminum pan was used as reference) with a heating rate of $10^{\circ} \mathrm{C} / \mathrm{min}$ under nitrogen atmosphere (at $50 \mathrm{~mL} / \mathrm{min}$ flow rate of nitrogen).

\section{Scanning Electron Microscopy (SEM)}

The SEM pictures of ASP-I and the generated crystals (ASP-II and ASP-III) were obtained using a scanning electron microscope (Jeol model no. JSM-6100, Japan). Samples were mounted on metal stub with double-sided adhesive tapes, and the samples were coated with gold under vacuum prior subjected into SEM observation.

\section{In Vitro Dissolution}

A membrane-free dissolution model as shown previously for oil-in-water nanosized emulsion (13) and PEG-based microparticles (14) was adapted in the current investigation to study the in vitro dissolution of different ASP crystals. The in vitro dissolution was performed in a water bath maintained at $37^{\circ} \mathrm{C}$ using $30 \mathrm{ml}$ vials in which $20 \mathrm{ml}$ of phosphate buffer solution of pH 6.8 (containing $0.005 \mathrm{ml}$ of span-80) was served as medium under sink condition. ASP-I, ASP-II, and ASP-III (unsieved crystals $5 \mathrm{mg}$ each) were kept in three separate vials. Periodically, $2 \mathrm{ml}$ of the sample solution was withdrawn from each vial at a time interval of 10, 20, 30, 40, 50, and $60 \mathrm{~min}$ with replenishment using $2 \mathrm{ml}$ fresh phosphate buffer solution of $\mathrm{pH}$ 6.8. The withdrawn samples after suitable dilution were analyzed using a UV-VIS spectrophotometer (model 200-20, Hitachi, Japan) to calculate the cumulative percentage drug dissolved values for different ASP crystals under these studied experimental conditions.

\section{RESULTS AND DISCUSSION}

Aspirin or acetyl salicylic acid (IUPAC name: 2acetoxybenzoic acid) is one of the most widely used pain killer, anticoagulant, antirheumatic, antiinflammatory, and antipyretic drugs. However, increasing amount of literature is also available either to describe the precipitation behavior of this drug during pre- and post-formulation stages or to illustrate the conversion from amorphous to crystalline or vice versa within the dosage form itself. Polymorphism of aspirin crystals has recently become an important issue, following reports of a second polymorph by Vishweshwar et al. (15) and Almarsson et al. (16). Although the two different polymorphs (form I and form II) of ASP crystals have been existed, the mysterious metastable form II (but pure and therapeutically effective one) has a tendency to grow within the crystals of stable form I as intergrowths (17). Furthermore, the form II crystals can be obtained by lyophilization (18), from water (19), rapid quenching of hot acetonitrile solution, or slow evaporation at ambient conditions (17). In addition, Bond et al. (20) and Bag and Reddy (21) have shown that the addition of aspirin anhydride from 2 to $10 \%$ to the crystallization solution of aspirin in acetonitrile or tetrahydrofuran enhances the formation of pure form II crystals. Recently, Jafari et al. (22) have also investigated the capability of the gas antisolvent (GAS) process to produce the therapeutically effective aspirin particles by studying the effect of four operating parameters namely, antisolvent addition, process temperature, solute concentration, and solvent type (methanol and acetone) on the final product particle size distribution, morphology, and crystallinity. Furthermore, the elusive form II of aspirin has been obtained during co-crystallization experiments of aspirin with levetiracetam or acetamide in acetonitrile after heating this solution at $70{ }^{\circ} \mathrm{C}$ followed by slow evaporation of this homogeneous solution over 2 days $(15,16)$. Collectively, all these studies indicated that depending on the experimental conditions applied at the time of their screening/ preformulation stage, the formation of pure or therapeutically effective form of crystalline API might have been occurred. To legally secure all such possible drug forms relatively at a shorter experimental duration/time in pharma industries, a new and efficient screening method was being developed with slight modification of the classical vapor diffusion method. The mechanisms by which the ASP crystal formation induced by DEE or DCM would however be the interest of synthetic chemical scientist, so, this part will not be discussed in detail here. Further works are being undertaken in our laboratory to see the additional controls such as comparison between crystals generated by the classical vapor diffusion method and studied method to demonstrate effectiveness (of reduced experimental time) of the suggested modified method without modification of the crystal internal structure. Therefore, it should be added that no attempt has been made in the present study to compare the crystals generated by the classical- and the modified vapor diffusion methods. Rather, an attempt was made to assess the feasibility of the modified vapor diffusion method in generating the drug crystals without modification of the crystal internal structure. And then, the influence of the selected two different non-solvents (DEE and DCM) on the drug crystal formation was studied followed by the characterization of the generated drug crystals using various analytical techniques. The aspirin crystal which was originally obtained in an unprocessed condition and used further to generate the other form of crystals is named as ASP-I. It should be mentioned that according to the manufacturer's disclosure, ASP-I was synthesized using acetic anhydride and salicylic acid, and the purification of ASP-I was done by crystallization with the addition of aspirin anhydride from 2 to $10 \%$ to the crystallization solution of aspirin in acetonitrile or tetrahydrofuran. The drug crystal which was generated by using DEE as the non-solvent is termed as ASP-II while the crystal generated after using DCM as the non-solvent is designated as ASP-III. In the present study, the results obtained from the various analytical techniques for all of these three crystals (ASP-I, II, and III) were compared. In fact, the purpose of studying the non-solvent role was to determine its impact on the growth mechanism and crystal characteristics, which has been the subject of much research. In addition, the degree of crystallinity of precipitated fine particles is also important, because it has a significant impact on the crystal growth mechanism during the crystallization process as well as on the performances (drug dissolution and subsequent drug absorption) of the final API.

The FT-IR spectra of unprocessed aspirin (ASP-I) crystals, and ASP crystals generated with DEE (ASP-II) or with DCM (ASP-III) by the modified diffusion method are shown in Fig. 2. The spectra of all the samples were found to be similar as the major absorption bands in ASP-I and its crystal forms ASP-II and ASP-III appeared at the same wave numbers (2999, 2872, 


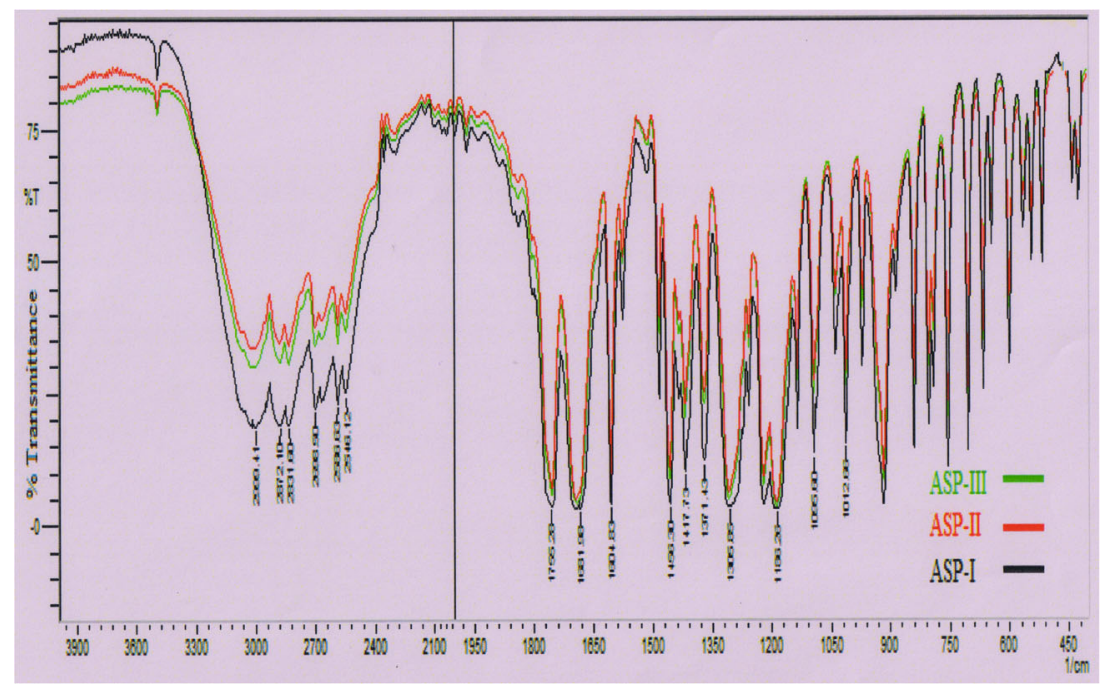

Fig. 2. Overlapped FT-IR spectra of unprocessed aspirin (ASP-I) crystals, and ASP crystals generated with diethyl ether $(A S P-I I)$ or with dichloromethane $(A S P-I I I)$ by the modified diffusion method

$1755,1604,1456,1305$, and $1186 \mathrm{~cm}^{-1}$ ). These results suggest that the internal structures of ASP-II and ASP-III crystals were identical when compared with ASP-I.

As previously mentioned, two non-solvents (namely, DEE and DCM) were used for the comparison of the degree of crystallinity of aspirin particles produced by the modified vapor diffusion method. Following $12 \mathrm{~h}$ refrigeration $\left(3-4{ }^{\circ} \mathrm{C}\right)$, the obtained crystals were washed with DEE or DCM, filtered, and then were allowed to dry in a hot air oven at $40^{\circ} \mathrm{C}$ for $10 \mathrm{~min}$. To study the influence of the drying condition $\left(40^{\circ} \mathrm{C}\right.$ for $\left.10 \mathrm{~min}\right)$ on the stability (form change if any) of the generated drug crystals, the drug crystals were simply kept under a fan at room temperature condition for $30 \mathrm{~min}$. Both the (hot air oven- and fan-dried) drug crystals were checked for their stability using X-ray crystallography. No significant differences were observed in the X-ray diffractograms of hot air oven-dried and fan-dried drug crystals (results not shown). This result thus ruled out the possible influence of the drying condition used on the drug crystal formed. The X-ray diffractograms of unprocessed aspirin (ASP-I) crystals, and ASP crystals generated with DEE (ASP-II) or with DCM (ASP-III) by the modified diffusion method are shown in Fig. 3. Peaks observed from the analysis were identical to those observed at diffraction angles of the reference pattern for aspirin as shown in the standard pharmacopoeias. The ASP-I crystal showed a sharp diffraction peaks at the following $2 \theta$ values and the corresponding relative intensity (RI) values which was given in parenthesis: $7.79(100 \%)$, 15.58 (75.19\%), 26.89 (11.73\%), 27.08 (8.30\%), and 31.41 $(12.18 \%)$. Similarly, the ASP-II crystals (generated by using DEE) consisted of diffraction peaks at $7.94(100 \%), 15.74$ (81.17\%), 26.93 (9.67\%), 27.24 (11.04\%), and 31.60 (9.62\%), whereas the ASP-III (generated by using DCM) showed the diffraction peaks at $7.76(31.74 \%), 15.63(100 \%), 26.82(4.27 \%)$, $27.12(2.84 \%)$, and $31.41(9.74 \%)$. From the X-ray diffractograms, it appears that all the three drug crystals exhibited the diffraction peaks at an almost the same $2 \theta$ values with only minor variations. Furthermore, no new peaks were found to appear in the diffractograms of the ASP-II and ASP-III crystals in comparison to the ASP-I crystal. The comparison of X-ray diffractograms thus ruled out the presence of different polymorphic forms for the drug crystals generated by the modified vapor diffusion method. However, the relative intensities of some of the diffraction peaks (especially at $2 \theta$ values of around 7.7 and 15.5) were found to be different. In fact, the relative intensity in the X-ray diffraction pattern explains the molecular arrangement of drug particles at each orientation. Furthermore, the difference observed in the peak intensities of the produced crystals may also be due to the impact of the non-solvent on the molecular arrangement during crystallization/precipitation. Thus, it is reasonable to speculate that the observed differences in the relative intensities of the peaks in the diffractograms of the ASP-II and ASP-III crystals in comparison

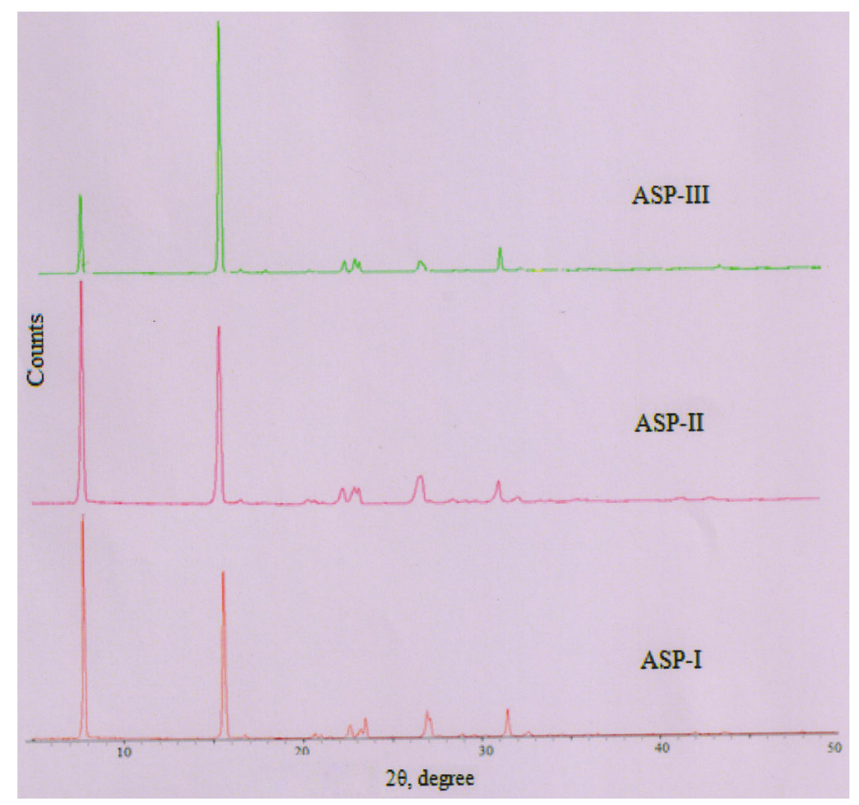

Fig. 3. XRD spectra of unprocessed aspirin (ASP-I) crystals, and ASP crystals generated with diethyl ether $(A S P-I I)$ or with dichloromethane $(A S P-I I I)$ by the modified diffusion method 
to the ASP-I crystal could be attributed to the crystal habit or crystal size modification $(23,24)$. In addition, this type of diversity observed in X-ray diffractograms by various other research groups have also been related either to the interaction between the solvent and the growing crystal surface or to the presence of solvent/non-solvent in the solute lattice $(25,26)$.

The DSC thermograms of unprocessed aspirin (ASP-I) crystals, and ASP crystals generated with DEE (ASP-II) or with DCM (ASP-III) by the modified diffusion method are shown in Fig. 4. Appearance of single sharp endothermic peak at around $137-139^{\circ} \mathrm{C}$ indicated the elimination of the occurrence of solid state transitions within the studied crystals (27). A similar sharp endothermic peak was also obtained by Bag and Reddy (21) while making the aspirin crystals by fast evaporation of solvent from DCM- or DEE-based drug solution using rotary evaporation techniques. Interestingly, by optimizing the solvent and temperature conditions, these authors have reported the selective preparation of aspirin forms I (stable) or II (kinetic) crystals starting from a same commercial sample (21). Starting from the same commercial sample (ASP-I), it could be of clinical interest to see whether or not the currently studied modified vapor diffusion method is able to increase the proportion of kinetic polymorph II in the stable polymorph I. According to the Ostwald's rule of stages (28), the kinetic polymorphs should crystallize first, then transform successively to more stable polymorphs when left in solution. Since 12 -h processing time was taken by the studied modified vapor diffusion method to generate ASP crystals, it also becomes necessary to confirm whether the solventmediated transformation from kinetic to stable polymorphic forms is occurring or not. Nevertheless, considering the highly volatile non-solvents such as DEE and DCM were used in the studied modified vapor diffusion method, one can speculate that the temperature control might play an important role to control the solvent-mediated transformation/conversion of

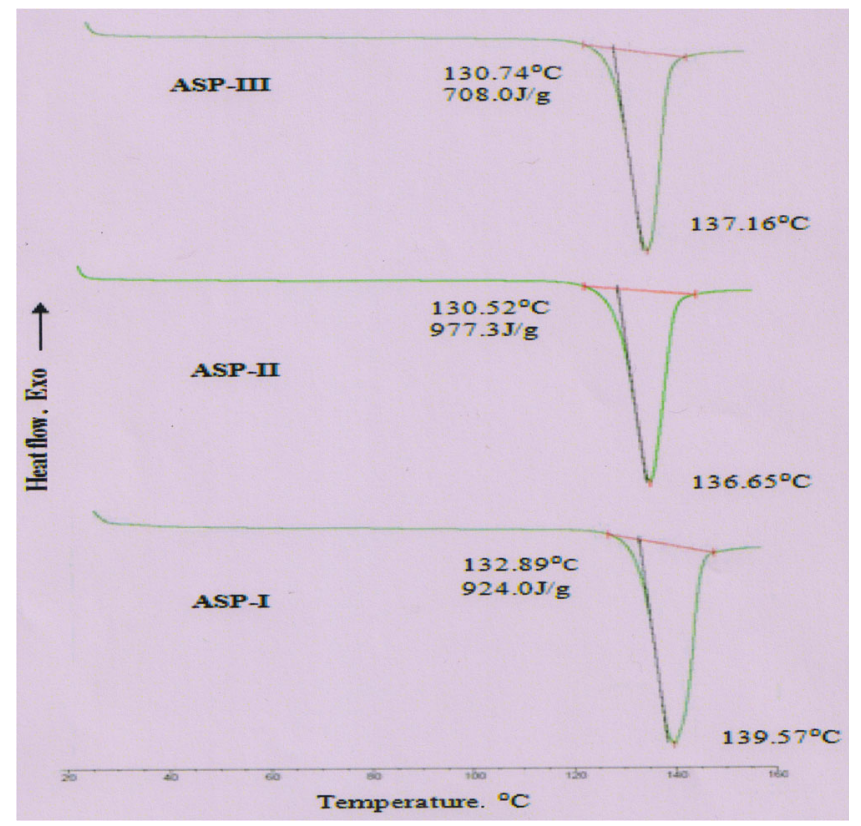

Fig. 4. DSC thermograms of unprocessed aspirin $(A S P-I)$ crystals, and ASP crystals generated with diethyl ether $(A S P-I I)$ or with dichloromethane $(A S P-I I I)$ by the modified diffusion method kinetic to stable polymorphic forms. Further optimization of experimental conditions are currently undergoing at our laboratory on how to prepare ASP crystals with high proportion of kinetic polymorph II in the stable polymorph I by the modified diffusion method.

Nevertheless, the observed single sharp endotherm further advocates that all the three tested crystals were of pure quality and the generated crystals (ASP-II and ASP-III) were indeed not solvates as no peak in the region prior to melting peak was observed $(29,30)$. Furthermore, the observed absence of a secondary peak in the DSC thermograms indicates/proves that the solvent content in the drug crystals was negligible, which was in agreement with the FDA guidelines for the solvent residual minimization in pharmaceutical products. In addition, an almost same onset of melting in the generated drug crystals ruled out the existence of polymorphic form II in the polymorphic form I. In addition, an almost same onset of melting in the generated drug crystals ruled out the existence of polymorphic form II in the polymorphic form I. The melting point of aspirin polymorph form II is ranged from 128 to $130^{\circ} \mathrm{C} \mathrm{(16)} \mathrm{and} \mathrm{for} \mathrm{aspirin} \mathrm{polymorph} \mathrm{form} \mathrm{I}$, it is $137^{\circ} \mathrm{C}$ (21). Since both of the non-solvents-induced ASP crystals have also showed the peak melting point value at an almost similar temperature $\left(136.65\right.$ and $137.16^{\circ} \mathrm{C}$, respectively, for ASP-II and ASP-III) close to the melting point value of aspirin polymorph form I, the DSC scans thus unequivocally speculates that only crystal habit modifications might occur but without involving any change in internal structure (23). This explanation is substantiated by the values of heats of fusion as shown for ASP-I, ASP-II, and ASP-III in Fig. 4. The heats of fusion value for ASP-II and ASP-III were -977.3 and $-708.0 \mathrm{~J} / \mathrm{g}$, respectively, while the heat of fusion value for unprocessed ASP-I was $-924.0 \mathrm{~J} / \mathrm{g}$. The increase and decrease in the heat of fusion values observed, respectively, for ASP-II and ASP-III from the heat of fusion value of ASP-I should explain the diversity in the degree of crystallinity of the drug crystals generated in the presence of two different nonsolvents in the modified vapor diffusion method.

The SEM pictures of unprocessed aspirin (ASP-I) crystals, and ASP crystals generated with DEE (ASP-II) or with DCM (ASP-III) by the modified diffusion method are shown in Fig. 5. It is clear from the micrographs that the modified vapor diffusion method has led to significant differences in the shape and appearance of crystals. ASP-I was found to be irregular-shaped crystals with no specific habit. However, ASP-II and ASP-III appeared to be platy and needle shaped, respectively. In the present study, it is observed that using of different non-solvents in the same crystallization process yielded different crystal habits. These differences in the crystal habit could be attributed to the non-solvent-surface-solvent interactions which lead to relative growth of the surfaces in different directions to produce a specific shape $(25,26)$.

In order to compare and/or substantiate the crystal habits, one of the most important experiments is dissolution study, and indeed, the dissolution slow down will impact in vivo behavior also. Figure 6 depicts the cumulative percentage drug dissolved from different ASP crystals in phosphate buffer solution of $\mathrm{pH} 6.8$ over the dissolution time period of 60 min. The pure drug (ASP-I) attained more than 100\% cumulative percentage drug dissolved value within $40 \mathrm{~min}$. The drug crystals generated in the presence of the non- 


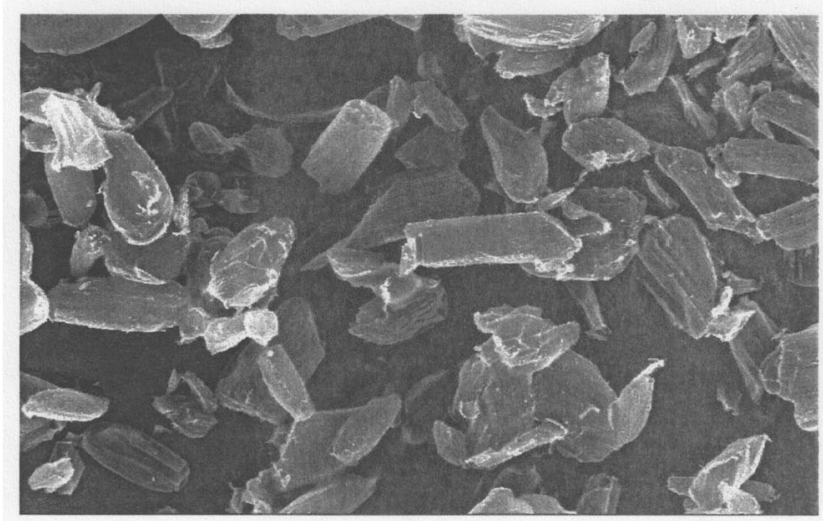

ASP-I

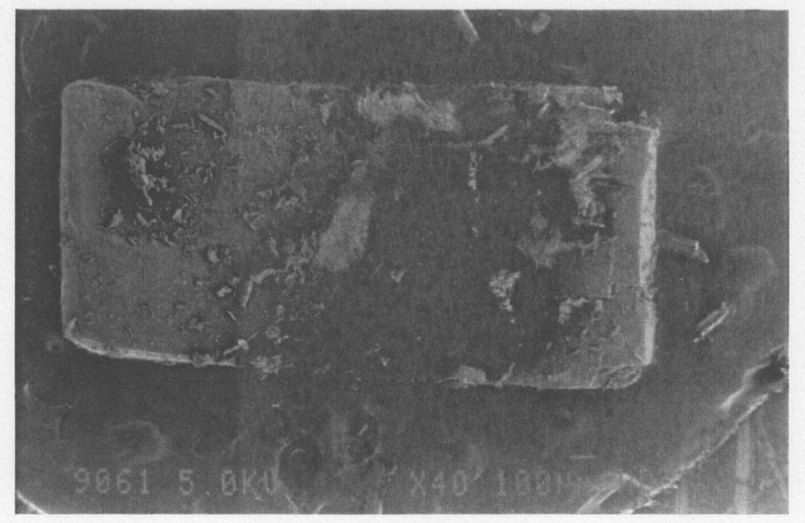

ASP-II

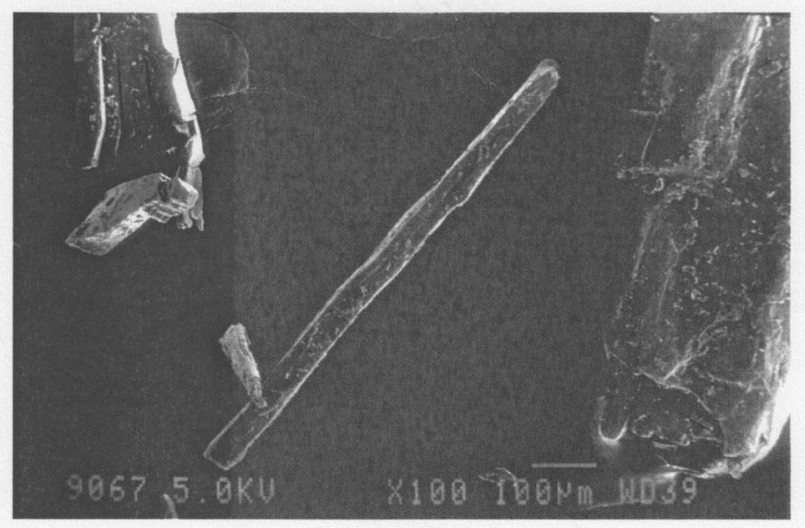

ASP-III

Fig. 5. Scanning electron micrographs of unprocessed aspirin $(A S P-I)$ crystals, and ASP crystals generated with diethyl ether $(A S P-I I)$ or with dichloromethane $(A S P-I I I)$ by the modified diffusion method

solvents DEE (ASP-II) and DCM (ASP-III) exhibited the cumulative percentage drug dissolved values that were significantly lesser than the cumulative percentage drug dissolved values obtained with ASP-I crystals at all of the studied dissolution time periods. When looking for the reason why the ASP-II and ASP-III exhibited the lesser cumulative percentage drug dissolved value than ASP-I, a recent patent by Almarsson et al. (16) disclosed that crystalline polymorphs typically have different solubilities from one another, and that a more thermodynamically stable polymorph is less soluble

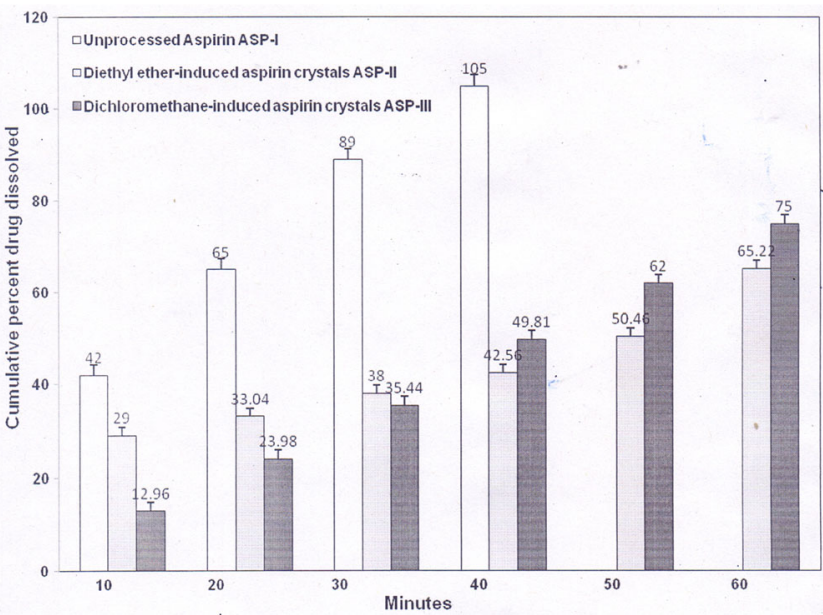

Fig. 6. Cumulative percentage drug dissolved from different aspirin $(A S P)$ crystals in phosphate buffer solution of $\mathrm{pH} 6.8$ over the dissolution time period of $60 \mathrm{~min}$

than a less thermodynamically stable polymorph. According to this general fact, both of the non-solvents-induced ASP (ASP-II and ASP-III) crystals are belonging to the more thermodynamically stable polymorph form I while the unprocessed ASP-I fits into the category of less thermodynamically stable polymorph form II. When looking at in vitro dissolution results, both of the non-solvents-induced ASP (ASP-II and ASP-III) crystals were, however, shown a peculiar drug dissolution pattern. At $10 \mathrm{~min}$ post-dissolution time period, the ASP-II crystals possessed the drug dissolved value of 29.00 $\pm 0.56 \%$ while the ASP-III crystals achieved the drug dissolved value of $12.96 \pm 0.72 \%$ only. Gradual increases in the cumulative percentage drug dissolved values were seen for 20 and 30 min post-dissolution time periods for both ASP-II and ASP-III crystals. Even, an almost juxtaposition in the cumulative percentage drug dissolved values was observed for ASPII $(38.00 \pm 0.88)$ and ASP-III $(35.44 \pm 0.55)$ crystals at $30 \mathrm{~min}$ post-dissolution time period. But after 30 min post-dissolution time period, the ASP-III crystals produced the cumulative percentage drug dissolved values that were higher than the cumulative percentage drug dissolved values shown by ASPII. Finally, at $60 \mathrm{~min}$ post-dissolution time period, the cumulative percentage drug dissolved values of $65.22 \pm 1.11$ and $75.00 \pm 1.08$ were observed, respectively, for ASP-II and ASPIII crystals. Collectively, the presence of two different nonsolvents in the modified vapor diffusion method resulted in the generation of ASP crystals that were shown two different cumulative percentage drug dissolved values within the studied dissolution time period of $60 \mathrm{~min}$. Further experimental works are currently undergoing at our laboratory to find out exactly the reason for the peculiar cumulative percentage drug dissolved values observed with ASP-II and ASP-III in phosphate buffer solution of $\mathrm{pH} 6.8$.

\section{CONCLUSION}

In contrast to the classical vapor diffusion method that entails many days for generating the API crystals, the modified vapor diffusion method which was shown in the present study requires only $12 \mathrm{~h}$ to generate the same upon screening 
with two different non-solvents (DEE and DCM). Two aspirin crystals (ASP-II and ASP-III) were generated from the unprocessed ASP-I by the modified vapor diffusion method upon screening with two different non-solvents. With a lone exception of FT-IR result, the X-ray diffraction and DSC results did show evidence of distinguishing features like the crystal habit or crystal size modification for the generated drug crystals in comparison with its original unprocessed crystal. The SEM pictures clearly indicate that the modified vapor diffusion method generated the drug crystals with different morphologies depending on the non-solvents employed. This observation is further substantiated with the results of dissolution study wherein the ASP-II and ASP-III crystals possessed a dissolution behavior that was slower than the dissolution behavior shown by the ASP-I crystals. Therefore, the modified vapor diffusion method could routinely be adapted as a screening method to generate all of the possible forms of the API for choosing the particular crystals with desired preformulation characteristics.

\section{ACKNOWLEDGMENTS}

The authors wish to thank Lovely Professional University (LPU), Phagwara, Punjab, India for providing the financial assistance to conduct this research and Central Instrumentation Laboratory (CIL), Panjab University Chandigarh, Punjab, India for allowing us to perform the various analytical instrumentation works.

\section{Compliance with Ethical Standards}

Declaration of Interest. All the authors have read and approved this version of the article and the authors report no declarations of interest.

\section{REFERENCES}

1. Haleblian JK. Characterization of habits and crystalline modification of solids and their pharmaceutical applications. J Pharm Sci. 1975;64:1269-88.

2. Garekani HA, Ford HA, Rubinstein MH, Rajabi-Siahboomi AR. Highly compressible paracetamol-II. Compression properties. Int J Pharm. 2000;208:101-10.

3. Tiwary AK. Modification of crystal habit and its role in dosage form performance. Drug Dev Ind Pharm. 2001;27:699-709.

4. Rasenack N, Müller BW. Crystal habit and tableting behavior. Int J Pharm. 2002;244:45-57.

5. Garekani A, Ford JL, Rubinstein MH, Rajabi-Siahboomi AR. Formation and compression characteristics of prismatic polyhedral and thin plate-like crystals of paracetamol. Int J Pharm. 1999;187:77-89.

6. Liu YJ, Wang J, Yin Q. The crystal habit of ciprofloxacin hydrochloride monohydrate crystal. J Cryst Growth. 2005;276:237-42.

7. Garekani A, Ford JL, Rubinstein MH, Rajabi-Siahboomi AR. Highly compressible paracetamol: I: crystallization and characterization. Int J Pharm. 2000;208:87-99.

8. Nokhodchi A, Bolourtchian N, Dinarvand R. Dissolution and mechanical behaviors of recrystallized carbamazepine from alcohol solution in the presence of additives. J Cryst Growth. 2005;274:573-84.
9. Mirmehrabi M, Rohani S, Murthy KSK, Radatus P. Polymorphic behavior and crystal habit of an anti-viral/HIV drug: stavudine. Cryst Growth Des. 2006;38:141-9.

10. Hilfiker R. Polymorphism: in the pharmaceutical industry. Weinheim: Wiley-VCH Verlag GmbH \& Co. KGaA; 2006.

11. Bag PP, Patni M, Reddy CM. A kinetically controlled crystallization process for identifying new co-crystal forms: fast evaporation of solvent from solutions to dryness. Cryst Eng Comm. 2011:13:5650-2.

12. Guillory JK. Generation of polymorphs, hydrates, solvates and amorphous solids. In: Brittain HG, editor. Polymorphism in pharmaceutical solids. New York: Marcel Dekker, Inc; 1999. p. 183226.

13. Tamilvanan S, Kumar BA. Influence of acetazolamide loading on the (in vitro) performances of non-phospholipid-based cationic nanosized emulsion in comparison with phospholipid-based anionic and neutral-charged nanosized emulsions. Drug Dev Ind Pharm. 2011;37:1003-15.

14. Tamilvanan S, Vinaykumar DS, Ashutosh T. In vitro evaluation of polyethylene glycol-based microparticles containing azithromycin. Drug Del Translat Res. 2014;4:139-48.

15. Vishweshwar P, McMahon J, Oliveira M, Peterson ML, Zaworotko MJ. The predictably elusive form II of aspirin. J Am Chem Soc. 2005;127:16802-3.

16. Almarsson $\mathrm{O}$, McMahon J, Vishweshwar P, Peterson M, Zaworotko MJ. Novel polymorph of acetylsalicylic acid, and methods of making and using the same. US Patent Publication Number: US2010/0331285 A1, Publication Date: Dec 302010.

17. Bond AD, Boese R, Desiraju GR. On the polymorphism of aspirin: crystalline aspirin as intergrowths of two polymorphic domains. Angew Chem Int Ed. 2007;46:618-22.

18. Tang X, Pikal M. Design of freeze-drying processes for pharmaceuticals: practical advice. J Pharm Res. 2004;21:191-200.

19. Sperger D, Chen B, Offerdahl T, Hong S, Schieber L, Lubach J, Barich D, Munson E. Characterization of a new form of aspirin. AAPS J. 2006; 8:13961, www.aapsj.org/abstracts/AM_2006/ AAPS2006-002764.pdf.

20. Bond AD, Solanko KA, Parsons S, Redder S, Boese R. Single crystals of aspirin form II: crystallization and stability. Cryst Eng Comm. 2011;13:399-401.

21. Bag PP, Reddy CM. Evaporation method: a case study of aspirin, anthranilic acid and niflumic acid. Cryst Growth Des. 2012;12:2740-3.

22. Jafari D, Yarnezhad I, Nowee SM, Baghban SHN. Gasantisolvent (GAS) crystallization of aspirin using supercritical carbon dioxide: experimental study and characterization. Ind Eng Chem Res. 2015;54:3685-96.

23. Garekani HA, Sadeghi F, Badiee A, Mostafa SA, RajabiSiahboomi AR. Crystal habit modifications of ibuprofen and their physicomechanical characteristics. Drug Dev Ind Pharm. 2001;27:803-9.

24. Panchagnula R, Sundaramurthy P, Pillai O, Agrawal S, Raj YA. Solid-state characterization of mefenamic acid. J Pharm Sci. 2004;93:1019-29.

25. Stoica C, Verwer P, Meekes H, Van Hoof PJCM, FM K s, Vlieg E. Understanding the effect of a solvent on the crystal habit. Cryst Growth Des. 2004;4:765-8.

26. Tiwary AK. Crystal habit changes and dosage form performance. In: Swarbrick J, editor. Encyclopedia of pharmaceutical technology. 3rd ed. New York: Informa Healthcare; 2006. p. 822.

27. Marjo CE, Bhadbhade M, Hook JM, Rich AM. Polymorphism and a metastable solvate of duloxetine hydrochloride. Mol Pharm. 2011;8:2454-64.

28. Ostwald WFZ. Physical chemistry. Stoechiom Verwandtschaftsl. 1897;22:289-302.

29. Giron D. Applications of thermal analysis in the pharmaceutical industry. J Pharm Biomed Anal. 1986;4:755-70.

30. Chadha R, Gupta S, Shukla G. Crystal habit, characterization and pharmacological activity of various crystal forms of arteether. Acta Pharm Sinica. 2011;B1:129-35. 